\title{
Attomicroscopy: attosecond electron microscopy
}

\author{
M. Th. Hassan
}

M. Th. Hassan, "Attomicroscopy: attosecond electron microscopy," Proc. SPIE 10753, Ultrafast Nonlinear Imaging and Spectroscopy VI, 107530R (3

September 2018); doi: 10.1117/12.2309426

EDent: SPIE Optical Engineering + Applications, 2018, San Diego, California, United States 
Invited Paper

\title{
Attomicroscopy: Attosecond Electron Microscopy \\ M. Th. Hassan \\ Department of Physics, University of Arizona, Tucson, Arizona 85721, USA
}

\begin{abstract}
The development of Ultrafast Electron Microscopy (UEM) and diffraction (UED) permit the imaging of atomic motion in real time and space. UEM and UED have found a vast range of applications spans chemistry, physics, material science, and biology. The temporal resolution in ultrafast electron imaging, typically on the order of few hundred femtoseconds, is limited by the electron pulse duration and the space charge effect. Hence, imaging the fast motions of electrons remains beyond the reach. Recently, we break the temporal resolution limits in UEM by generating a 30-fs electron pulse exploiting the optical gating approach. The gating technique is based on the electron-laser coupling where the free electrons in the wavepacket exchange energy with the light photons of the laser pulse. In this case, the optical laser pulse acts as a temporal gating for the electrons which gain/loss energy. These electrons are filtered out and generate an electron pulse with a temporal profile similar to the gating laser pulse. The obtained, few tens of femtosecond, temporal resolution opens the door- for the first time- to image the electron dynamics in real time. Moreover, the optical attosecond pulse, which has been demonstrated earlier, eventually will be used to gate the electrons in a sub-femtosecond time window. So, the gated electrons will generate a single isolated attosecond electron pulse. This unique tool will establish the attosecond electron imaging tool which we so-called "Attomicroscopy". Attomicroscopy will enable the imaging of the electron motion, last few hundreds of attosecond to few femtoseconds, in action.
\end{abstract}

Keywords: Attosecond electron pulse, attosecond electron microscopy, imaging the electron motion, ultrafast electron microscopy, femtosecond electron diffraction, optical gating.

\section{INTRODUCTION}

In the last century, the development of electron microscopy and X-ray diffraction afforded a remarkable tool for imaging and resolving the three-dimensional structure of matter with atomic resolution, which had a great impact in different fields [1, 2]. Recently, the fourth-dimension "time" has been introduced for probing matter dynamics, by utilizing electron bursts. The generation of ultrafast (picosecond and femtosecond) electron pulses enabled the establishment of Ultrafast Electron Diffraction (UED), Ultrafast Electron Microscopy (UEM), and Scanning Ultrafast Electron Microscopy (SUEM). These tools enabled the recording of images of the structure dynamics and atomic motion in real time and space. These tools have found numerous applications in chemistry, physics, biology, and materials science [3-9]. Currently, the research activities in the field focus on improving both the spatial and temporal resolutions in order to resolve the ultrafast dynamics of matter with better contrast. In ultrafast electron diffraction and microscopy experiments, the temporal resolution is defined by the electron pulse duration and its synchronization stability with the triggering optical pulses. However, the electron pulse suffers from a temporal broadening due to the space-charge effect and energy dispersion during its propagation from the source to the sample, which ruins both the temporal and spatial resolutions. Therefore, many electron-pulse compression techniques have been developed for controlling the space-charge effect and generating ultrashort (bright) electron pulses $[10,11]$. These techniques enable the confinement of the electron pulse to hundred femtoseconds; however, it suffers from time jittering and the temporal synchronization issues, which limit the temporal resolution response in time-resolved electron experiments. Therefore, the ultrafast dynamics measurements that have been carried out so far are in the timescale of picosecond to several hundreds of femtoseconds [12-16]. Therefore, the imaging of faster dynamics (i.e. electron dynamics) in matter still remains beyond reach.

Lately, we demonstrated the generation of the shortest electron pulse (30 fs) in UEM by the optical gating approach, which breaks the conventional compression limits of an electron pulse and attains electron dynamics temporal resolution in

Ultrafast Nonlinear Imaging and Spectroscopy VI, edited by Zhiwen Liu, Demetri Psaltis, Kebin Shi, Proc. of SPIE Vol. 10753, 107530R · @ 2018 SPIE · CCC code: 0277-786X/18/\$18 · doi: 10.1117/12.2309426 
electron microscopy [17]. In the optical gating approach, the generated gated electron pulse duration is limited only by the gating laser pulse, which could be on the attosecond time scale [18]. The optical gating using the laser attosecond pulse spans over two octaves visible and flanking spectral regions would lead to generate the isolated attosecond electron pulses and open the way for establishing the "Attomicroscopy" [17]. This new tool will allow the real-time imaging of electronic motion as theoretically studied in atoms, molecules [19], and condensed matter [20], which could radically change our insight into the workings of the microcosm and could hold the promise for breaking new grounds in a number of fields of science and technology.

\section{OPTICAL GATING OF ULTRAFAST ELECTRON PULSE}

The principle of the optical gating approach (illustrated in Figure 1) can be explained as follows [17, 21]; free electrons and laser (photons) pulses interact with inelastic scattering on a nanostructure [21,22]. This interaction causes the electrons to gain (or lose) a single quantum or multiple photon quanta. Therefore, photon-electron coupling results in some electrons (in the electron pulse) gaining or losing one or more photon quanta - but only in the presence of the optical laser pulse. Hence, the optical pulse is a temporal optical gate for these electrons that have a temporal profile similar to the gating window (i.e., the optical laser pulse duration). Then, these gated electrons - can be resolved as side peaks around the zeroloss peak in the electron energy spectra (Figure 1) —are filtered out to obtain ultrashort, attosecond-scale electron pulses. Such pulses will provide a significant enhancement to temporal resolution in UEM and UED for exploring the ultrafast dynamics of matter triggered by another ultrashort optical laser pulse [17, 23].

The optical gating approach has striking features over the conventional electron pulse compression techniques [23], these features are ; (i) the temporal gating window and duration of the generated electron pulse is limited only by the optical gating pulse, (ii) the phase drift between the optical gating pulse (and consequently gated-electron pulse) and the laser pulse triggering dynamics would also on the attosecond time scale, thanks to the optical phase locking technique, and (iii) the gated electron pulses have enough intensity to practically image sample dynamics since the original electron pulse is not restricted to be in the single electron-regime because the gated electron pulse is generated after acceleration and at the position of the sample under study. Therefore, this approach is a promising technique to break the limits of the temporal resolution not only in the ultrafast electron microscopy, but also for the free-electron lasers, where extreme time-resolution is difficult to achieve.

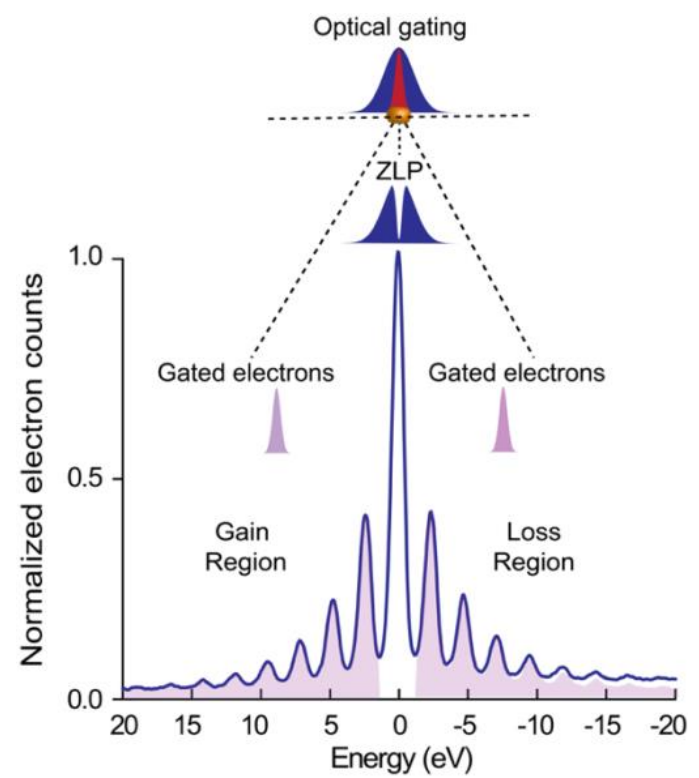

Figure. 1. Optical gating of electron pulses. The few femtosecond electron pulses interact with the gating laser pulses on nanostructure. The gated electrons are presented in the electron energy spectrum as multiple peaks in the both sides of the zero-loss-peak. The gated electron spectral peaks are separated by one or multiple photon quanta of the gating laser pulse. 


\subsection{Generation of $30 \mathrm{fs}$ electron pulse by optical gating}

Exploiting the optical gating approach, we utilized ultrashort visible laser pulse (30 fs) to gate electrons in the wavepackets of few hundred femtosecond electron pulses generated by the photoemission of a photocathode and accelerated inside the ultrafast electron microscope [17]. Some electrons in the wavepackets exchange energy with the laser photons. The exchange happened in a time scale similar to the duration of the laser pulse and generate, to our knowledge, the shortest (30 fs) isolated electron pulse inside UEM. Accordingly, the temporal resolution in UEM is enhanced by more than an order of magnitude (16 times). This advancement permits the resolution and imaging of fast atomic motion, and the electron dynamics occur on the scale of a few tens of femtoseconds.

This optical gating experiment (setup is illustrated in Figure 1) can be briefly explained as follows; a train of IR (350 fs$120 \mu \mathrm{J})$ laser pulses $(\lambda \sim 1040 \mathrm{~nm}, 100 \mathrm{kHz}$ repetition rate) is divided into three beams, with $5 \%$ going through a second harmonic process twice to generate ultraviolet (UV) laser pulses $(\lambda \sim 260 \mathrm{~nm})$. These UV pulses are directed to the photoemissive cathode inside the microscope to generate ultrafast electron pulses (Original electron probe pulse), which are accelerated $(200 \mathrm{KeV})$ in the microscope column. The rest of the IR $(95 \%)$ is divided by a $50 / 50$ beamsplitter into two beams. Each beam enters into a different non-collinear optical parametric amplifier (NOPA) system. In the first one (NOPA-3H), the visible laser pulses are generated $(\lambda \sim 550 \mathrm{~nm})$. These pulses are compressed and have a FWHM pulse duration of $\sim 30$ fs. The second one (NOPA-2H) generates $\sim 33$ fs laser pulses in the near-IR (NIR) region $(\lambda \sim 740 \mathrm{~nm})$. The delays between the electron, visible, and NIR pulses are controlled by linear delay stages.

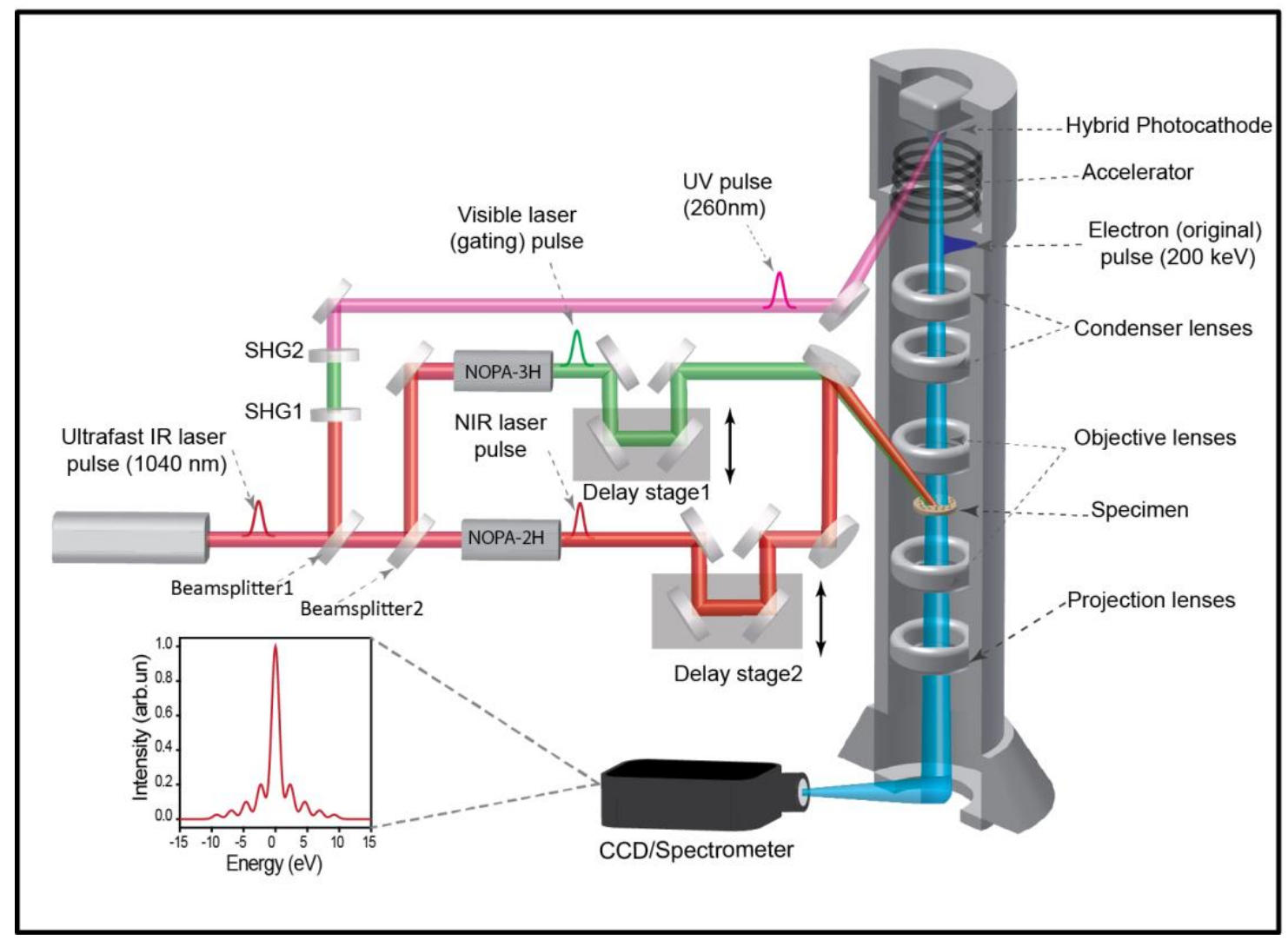

Figure. 2. Experimental setup of the optical-gating in UEM. A portion of infrared laser pulses generates ultraviolet (UV) laser pulses by two sequential second harmonic generation processes. These pulses are directed to the photoemissive cathode to generate ultrafast electron pulses. The remaining IR is divided into two equal beams sent to two NOPA systems to generate visible optical "gating" pulses ( $\sim 30 \mathrm{fs})$ and near-infrared (NIR) laser pulses $(\sim 33 \mathrm{fs})$. The delays between these pulses are controlled by linear delay stages. These pulses are recombined and focused onto the specimen in the microscope and the electron energy spectrum of the electron pulses is acquired by an electron energy spectrometer. An energy spectrum for electronvisible photon coupling is shown in the inset. 
The visible laser pulse acts as a "gating pulse" to generate a "gated" electron pulse emulating its pulse duration ( $\sim 30 \mathrm{fs})$, and the NIR pulse is utilized to characterize the temporal profile of the gated electron pulse. Notably, the two laser pulses (visible and NIR) are precompensated by a built-in prism compressor implemented inside each NOPA system for the dispersion accumulated due to the laser beams traveling through the air and glass up to the sample position. The correct compression was determined by introducing an amount of glass equivalent to beam splitters, lens, and microscope window before the autocorrelator apparatus, which was used to characterize the optical pulses temporal profile. Hence, we ensure that the "gating" visible laser pulse maintains its 30-fs pulse duration at the sample position inside the microscope where the optical gating process occurs. On the nanostructure sample, the "gating" visible pulse is kept at the maximum temporal overlapping with the electron pulse generated from the photocathode to obtain the optimum gating efficiency and generate the intense isolated "gated" ultrashort electron pulse.

\subsection{Temporal characterization of the 30-fs electron pulse}

The temporal profile of the gated electron pulse was characterized by the cross-correlation method. This occurred in two steps. First, the temporal profile of the original (few hundred femtoseconds) electron pulses, generated by photoemission from the photocathode inside the microscope, was characterized by cross-correlation between the "gating" visible pulse and the electron pulse. Since the "gating" pulse duration is much shorter, the cross-correlation profile directly reflects the "original" electron pulse temporal profile. The retrieved pulse duration of these "original" electron pulses is on the order of $500 \mathrm{fs}$. Second, the gated electron pulse was characterized by measuring cross-correlation temporal profile of the NIR (33 fs) laser pulse with the original and gated electrons. The cross-correlation spectrogram (figure 3(b)) was recorded. Then, the temporal profile of this cross-correlation was retrieved from the spectrogram, which carries the signature of the coupling between the original electron pulse and NIR laser pulse and that between the gated electron pulse and NIR laser

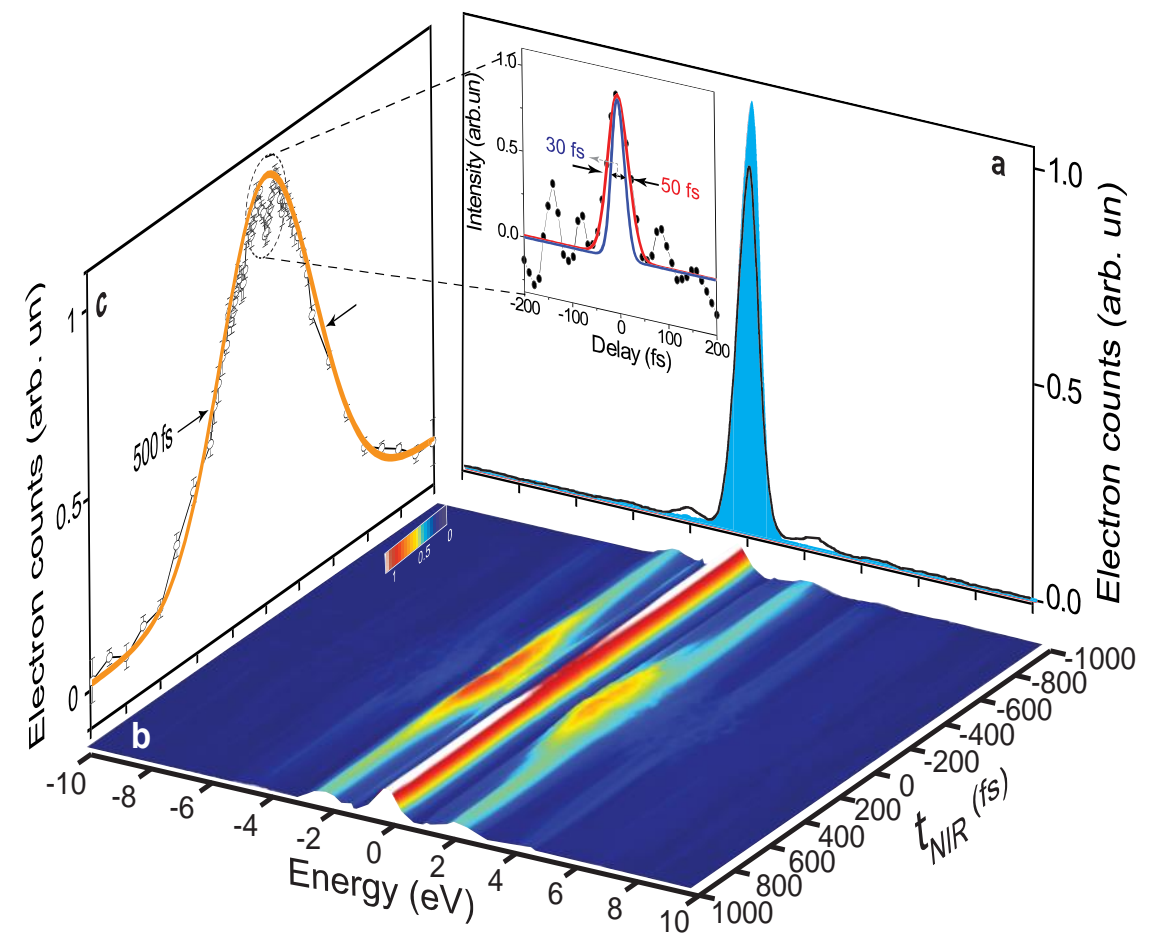

Figure 3. (a) The electron energy spectra represent the original electron pulse (ZLP) (blue shaded curve) and the coupling between "original" electron pulse and "gating" visible pulse (30 fs) (black line). (b) The cross-correlation electron energy spectrogram of the electron-photon coupling between the NIR laser pulse and both "original" and "gated" electron pulses. (c) The cross-correlation temporal profile retrieved from the measured spectrogram. inset in (a) shows the cross-correlation profile of the "gated" electron and NIR pulse (FWHM=50 fs) and the retrieved temporal profile of the generated gated electron pulse $(\mathrm{FWHM}=30 \mathrm{fs})$. 
pulse (figure 3(c)). The cross-correlation temporal profile of the last one is shown in the inset of figure 3(a), and it shows an FWHM on the order of $50 \mathrm{fs}$. Since the NIR pulse duration is $33 \mathrm{fs}$. Therefore, the gated electron pulse duration can be retrieved to be on the order of $30 \mathrm{fs}$, similar to the gating pulse temporal profile. The generated "gated" electron pulse has sufficient electron counts ( $\sim 8 \%$ of the total electron counts or $<1$ electron/pulse) for probing the ultrafast dynamics of matter. The attained temporal resolution (30 fs) allows the imaging of the electron dynamics, lasts a few tens of femtoseconds which was beyond reach before, such as the electron-electron scattering and electron-phonon coupling in semiconductors [24], and the dynamics of surface plasmons [25].

\section{ATTOMICROSCOPY: ATTOSECOND ELECTRON IMAGING}

\subsection{Single isolated attosecond electron pulse}

As mentioned earlier, the gated electron pulse duration depends on the temporal gating window, which is defined by the optical gating pulse duration. Therefore, the optical attosecond pulse (380 as)[18] would provide the sub-femtosecond temporal gating resolution desired to generate isolated attosecond electron pulses by the optical gating approach. Moreover, extreme phase stability ( $<1 \mathrm{fs}$ ) can be achieved between the pump and gating laser pulses by phase-locking. Both will enable to attain the attosecond temporal resolution in electron microscopy and establish the "Attomicroscopy". Here, we conducted a simple theoretical calculation, in which a compensated $10 \mathrm{fs}$ electron pulse is gated by optical attosecond pulses (380 as), demonstrated early in [18]. This was done by calculating the probability of one electron emitting or absorbing photons through interaction with the surface of a nanostructure [23]. Briefly, the electron-photon interaction is mediated by an evanescent electromagnetic field induced by the optical gating photon pulse hitting the surface of nanostructure (gating medium). The spatial distribution of the evanescent field is determined by the optical property and the geometry of the nanostructure. The attosecond optical gating of $10 \mathrm{fs}$ electron pulse allows the generation of isolated attosecond electron pulses (Fig. 4). For such extremely short-broadband "gating" optical pulse, the gating medium should have a broad frequency response (e.g. aluminium nanostructure, which has a Surface Plasmon lifetime in the attosecond time scale) and the gated electron spectral peaks are expected to exhibit energy broadening since the optical attosecond pulse spans over more than two octaves (spectral FWHM=1.75 eV) as shown in figures 4.

\section{(a)}

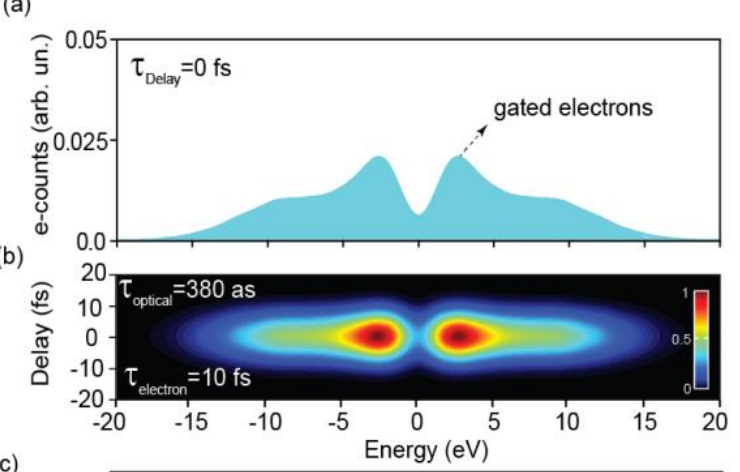

(c)

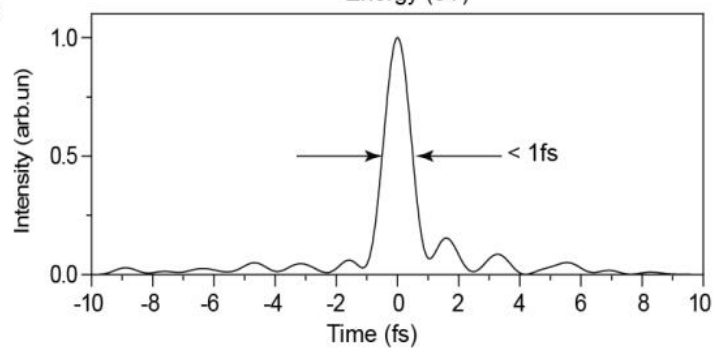

Figure 4. Attosecond optical gating of electron pulse. (a) Calculated spectrum of the gated electron of 10 fs electron pulse with OAP pulse centred at $530 \mathrm{~nm}$ at a delay time $=0$ fs. (b) Calculated full spectrogram from -20 to 20 fs. The zero-loss-peak is removed for better illustration. (c) Subfemtosecond temporal window and the temporal profile of the optical attosecond pulse to generate the isolated attosecond electron pulses by optical gating. 


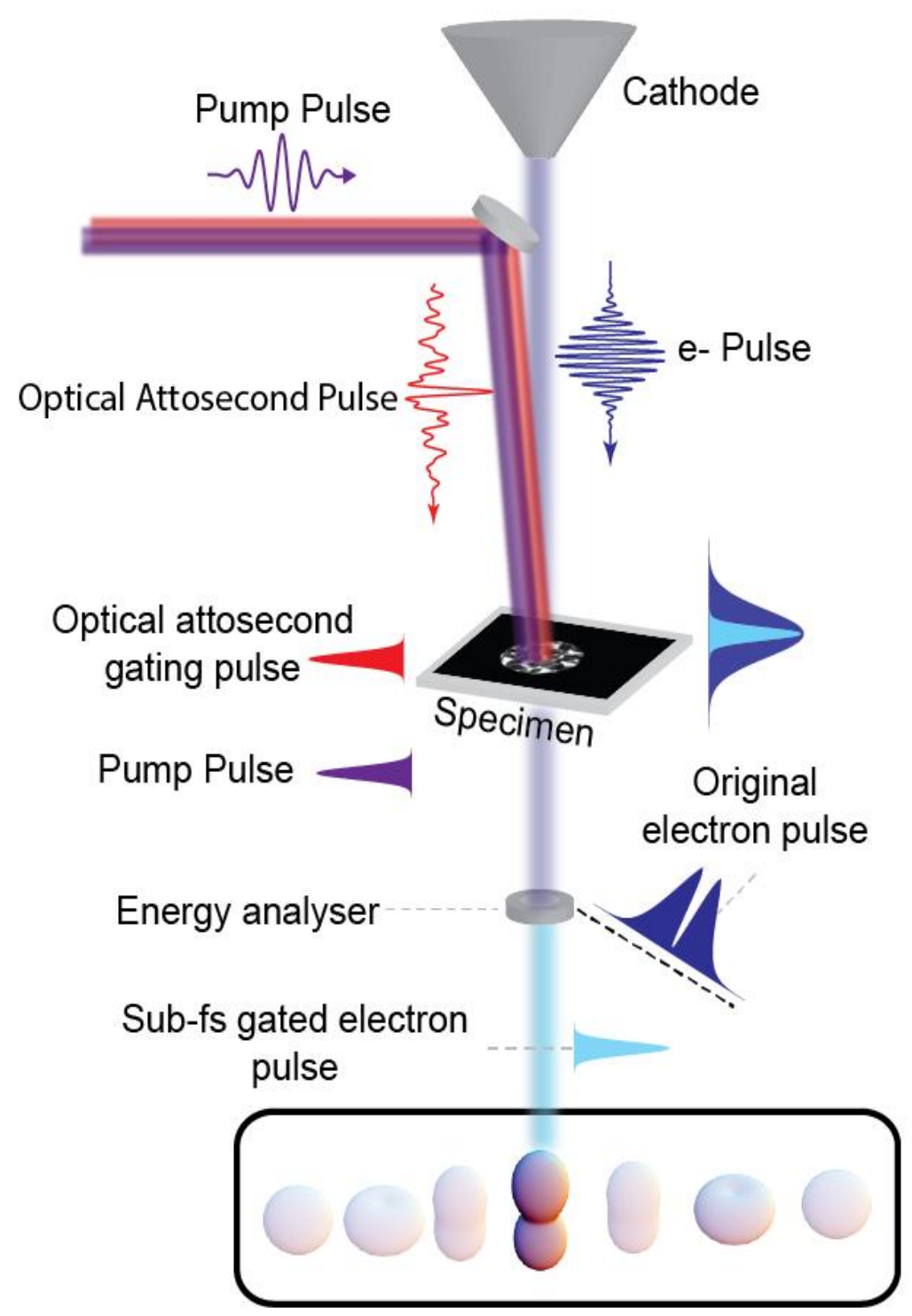

Figure 5. Attomicroscopy: Imaging the electron motion in real time. Illustration of Attomicroscopy setup. The optical attosecond pulse is used to provide the sub-femtosecond optical gating window for the original electron pulse in order to generate an isolated attosecond electron pulse. The optical gating and the generation take place at the sample stage. The generated gated pulse can be filtered out later, providing a desired probe resolution to image the electron motion in real time.

For the potential time-resolved Attomicroscopy experiment, the isolated attosecond "gated" electron pulse on the sample stage can be filtered out and utilized as a "probe" pulse, and another optical pulse can be utilized as a "pump" pulse to ultimately image the electron motion in real time. The isolated attosecond electron pulses can be generated and characterized right on the surface of the nanostructure sample which allows for enduring the attosecond resolution on the position where the experiments take place.

In conclusion, the generation of the isolated attosecond electron pulses by attosecond optical gating and the establishment of the Attomicroscopy open new avenues and allow for an enormous of femtosecond and attosecond electron imaging applications in different areas and could eventually enable the recording of a movie of electron motion in the act. 


\section{REFERENCES}

[1] M. Knoll and E. Ruska, "Das elektronenmikroskop," Zeitschrift für Physik, vol. 78, no. 5-6, pp. 318-339, 1932.

[2] J. D. Bernal and D. Crowfoot, "X-ray photographs of crystalline pepsin," Nature, vol. 133, no. 3369, pp. 794$795,1934$.

[3] A. H. Zewail, 4D Visualization of Matter. Imperial College Press, 2014.

[4] R. J. D. Miller, "Femtosecond Crystallography with Ultrabright Electrons and X-rays: Capturing Chemistry in Action," Science, vol. 343, no. 6175, pp. 1108-1116, 2014.

[5] G. Sciaini and R. D. Miller, "Femtosecond electron diffraction: heralding the era of atomically resolved dynamics," Reports on Progress in Physics, vol. 74, no. 9, p. 096101, 2011.

[6] R. D. Miller et al., "Making the molecular movie': first frames," Acta Crystallographica Section A: Foundations of Crystallography, vol. 66, no. 2, pp. 137-156, 2010.

[7] A. H. Zewail and J. M. Thomas, 4D Electron Microscopy: Imaging in Space and Time. Imperial College Press, 2010.

[8] A. H. Zewail, "Four-dimensional electron microscopy," Science, vol. 328, no. 5975, pp. 187-193, 2010.

[9] A. H. Zewail, "4D ultrafast electron diffraction, crystallography, and microscopy," Annual Review of Physical Chemistry, vol. 57, pp. 65-103, 2006.

[10] T. Van Oudheusden, P. Pasmans, S. Van Der Geer, M. De Loos, M. Van Der Wiel, and O. Luiten, "Compression of subrelativistic space-charge-dominated electron bunches for single-shot femtosecond electron diffraction," Physical Review Letters, vol. 105, no. 26, p. 264801, 2010.

[11] M. Gao et al., "Full characterization of RF compressed femtosecond electron pulses using ponderomotive scattering," Optics Express, vol. 20, no. 11, pp. 12048-12058, 2012.

[12] H. Ihee et al., "Direct imaging of transient molecular structures with ultrafast diffraction," Science, vol. 291, no. 5503, pp. 458-462, 2001.

[13] B. J. Siwick, J. R. Dwyer, R. E. Jordan, and R. D. Miller, "An atomic-level view of melting using femtosecond electron diffraction," Science, vol. 302, no. 5649, pp. 1382-1385, 2003.

[14] R. Ernstorfer, M. Harb, C. T. Hebeisen, G. Sciaini, T. Dartigalongue, and R. D. Miller, "The formation of warm dense matter: experimental evidence for electronic bond hardening in gold," Science, vol. 323, no. 5917, pp. 1033-1037, 2009.

[15] M. Gao et al., "Mapping molecular motions leading to charge delocalization with ultrabright electrons," Nature, vol. 496, no. 7445, pp. 343-346, 2013.

[16] V. R. Morrison et al., "A photoinduced metal-like phase of monoclinic VO2 revealed by ultrafast electron diffraction," Science, vol. 346, no. 6208, pp. 445-448, 2014.

[17] M. T. Hassan, J. S. Baskin, LiaoB, and A. H. Zewail, "High-temporal-resolution electron microscopy for imaging ultrafast electron dynamics," Nature Photonics, Article vol. 11, no. 7, pp. 425-430, 07//print 2017.

[18] M. T. Hassan et al., "Optical attosecond pulses and tracking the nonlinear response of bound electrons," Nature, Letter vol. 530, no. 7588, pp. 66-70, 02/04/print 2016.

[19] H.-C. Shao and A. F. Starace, "Detecting Electron Motion in Atoms and Molecules," Physical Review Letters, vol. 105, no. 26, p. 263201, 12/20/ 2010.

[20] V. S. Yakovlev, M. I. Stockman, F. Krausz, and P. Baum, "Atomic-scale diffractive imaging of sub-cycle electron dynamics in condensed matter," Scientific Reports, vol. 5, 2015.

[21] M. T. Hassan, H. Liu, J. S. Baskin, and A. H. Zewail, "Photon gating in four-dimensional ultrafast electron microscopy," Proceedings of the National Academy of Sciences, vol. 112, no. 42, pp. 12944-12949, October 20, 20152015.

[22] B. Barwick, D. J. Flannigan, and A. H. Zewail, "Photon-induced near-field electron microscopy," Nature, 10.1038/nature08662 vol. 462, no. 7275, pp. 902-906, 12/17/print 2009.

[23] M. T. Hassan, "Attomicroscopy: from femtosecond to attosecond electron microscopy," J. Phys. B: At. Mol. Opt. Phys., vol. 51, p. 032005, 2018.

[24] F. Rossi and T. Kuhn, "Theory of ultrafast phenomena in photoexcited semiconductors," Reviews of Modern Physics, vol. 74, no. 3, p. 895, 2002.

[25] M. I. Stockman, "Nanoplasmonics: past, present, and glimpse into future," Optics Express, vol. 19, no. 22, pp. 22029-22106, 2011/10/24 2011. 